\title{
MiR-149 attenuates the proliferation and migration of TGF- $\beta 1-$ induced airway smooth muscle cells by targeting TRPM7 and affecting downstream MAPK signal pathway
}

\author{
Zhengyu Zhu' ${ }^{1}$, Liya Zhang ${ }^{2}$, Ting Jiang33, Yan Qian³, Yun Sun ${ }^{3}$ and Qian Zhang ${ }^{3} \square$ \\ 1Department of Pediatrics, The Affiliated Changzhou No.2 People's Hospital of Nanjing Medical University, Changzhou City, Jiangsu Province, \\ 213000, China; 2Department of Emergency, The Affiliated Changzhou No.2 People's Hospital of Nanjing Medical University, Changzhou City, \\ Jiangsu Province, 213000, China; ${ }^{3}$ Department of Respiratory and Critical Care Medicine, The Affiliated Changzhou No.2 People's Hospital of \\ Nanjing Medical University, Changzhou City, Jiangsu Province, 213000, China
}

Asthma is considered as a general term for various chronic inflammatory diseases of the respiratory tract. Growing evidences have supported that microRNAs were involved in mediating cell proliferation, migration, and other cellular functions. MiR-149 has been found to take part in the development of various cancers. However, whether miR-149 participated in the proliferation and migration of transforming growth factor beta 1 (TGF- $\beta 1$ )induced airway smooth muscle cells was still unknown. In this study, the expression level of miR-149 in human airway smooth muscle cells (ASMCs) was decreased after TGF- $\beta 1$ treatment in vitro. Additionally, the over-expression of miR-149 obviously suppressed proliferation and migration in human ASMCs. Besides, we found that overexpression of miR-149 could inhibit the expression of transient receptor potential melastatin 7 (TRPM7) both in protein and gene levels. Furthermore, we demonstrated that miR-149 could inhibit the cell proliferation and migration in human ASMCs by targeting TRPM7 through modulating mitogen-activated protein kinases (MAPKs) signaling pathway. Taken together, we strongly supported that miR-149 might be a key inhibitor of asthma by targeting TRMP7. Therefore, our finding suggests a promising biomarker for the development of further targeted therapies for asthma.

Key words: MiR-149, human airway smooth muscle cells (ASMCs), TRPM7, MAPKs pathway

Received: 08 July, 2020; revised: 03 September, 2020; accepted: 11 October, 2020; available on-line: 07 December, 2020

『e-mail: Oian Zhang121@163.com

Acknowledgements of Financial Support: This study was supported by grants from the Jiangsu province social development project (BE2020651 to Q.Z.), and in part from the Jiangsu province "333 talents" project (BRA2020015 to Q.Z.), the Changzhou Sci and Tech Program (CE20205023 to Q.Z.), the Changzhou High-Level Medical Talents Training Project (2016CZLJ017 to Q.Z.).

Abbreviations: ASMCs, airway smooth muscle cells; MAPKs, modulating mitogen-activated protein kinases; TRPM7, transient receptor potential melastatin 7

\section{INTRODUCTION}

Bronchial asthma, commonly known as asthma, is a chronic respiratory disease with an increasing incidence rate over decades on a global scale (Kim et al., 2018). Currently, asthma has been considered as the main chronic disease threatening public health in the world. However, the pathogenesis of asthma is complex and has not been well elucidated. Chronic airway inflammation, which was demonstrated to be the essence of asth- ma, is one of the most important mechanisms leading to airway hyper-responsiveness in asthma (Chapman \& Irvin, 2015). Airway remodeling is also accompanied with chronic airway obstruction and asthma (Siddiqui et al., 2018). The cell types and airway components involved in the occurrence and development of airway remodeling in asthma are very complex. ASM cells, which acted as the contraction effect cells, are directly related to airway contracture and airflow limitation. The proliferation and migration of ASM cells is an important factor for airway remodeling in asthma. MicroRNA (miRNA), one type of short non-coding RNA molecules, is an endogenous non coding single stranded microRNA molecule with a length of 21-25 nucleotides (Bartel, 2004). Mature miRNAs are involved in modulating the cell proliferation, migration, apoptosis, metabolism and other cellular responses by directly degrading target gene mRNA or inhibiting its translation process to negatively regulate gene expression (Gan \& Denecke, 2013). In addition, microRNAs are also closely related to a variety of pathological processes such as inflammation, tumor, and angiogenesis. Meanwhile, microRNAs also participate in the occurrence of various system diseases including respiratory, digestive, nervous and urinary system (Lou et al., 2017). At present, the regulatory role of miRNA in asthma and airway remodeling has been revealed in several literatures (Weidner et al., 2019). However, there is no study to point out its specific role and potential mechanism (Hu et al., 2017). Therefore, it is a promising strategy to explore the function and mechanism of miRNAs to gain insight into the pathogenesis of asthma and to develop new biomarkers for the diagnosis and treatment of asthma.

The family of proteins with transient receptor potential (TRP) that are located in numerous cell membranes contains TRPM (melastatin), TRPV (vanilloid), TRPC (canonical), TRPP (polycystin), TRPML (mucolipin) and TRPA (subfamily A), is responsible for many cellular functions (Fleig \& Penner, 2004). Among them, the transient receptor potential melastatin 7 (TRPM7) has ion channel and kinase activities (Faouzi et al., 2017). It has been reported that TRPM7 was expressed in a variety of cancers (melanoma, human head and neck cancer, breast cancer, gastric cancer, etc.) and plays pivotal roles in regulating cell survival, apoptosis and cell cycle progression (Yee, 2017). It has also been demonstrated that the binding of TRPM7 to miR-28-5p resulted in the suppression of cell proliferation, migration and invasion in glioma cells (Wan et al., 2019). In addition, miR-543/ TRPM7 axis is also involved in the initiation and pro- 
gression of cervical cancer (Liu et al., 2019). Similarly, miR-129-3p is reported to directly target to TRPM7 and act as a negative regulator for TRPM7 expression in a renal cell clear cell carcinoma (Zhao et al., 2018). Although various studies have supported the importance of the interaction of TRPM7 and miRNA in cell proliferation, migration, and other cellular functions, whether the binding between TRPM7 and miR-149 plays an important role in the progression of asthma is still unknown. Transforming growth factor $\beta 1$ (TGF- $\beta 1$ ) has been (Ojiaku et al., 2017) proved to stimulate the cell proliferation of ASMCs in vitro.

In this study, we aimed to explore whether the interaction between TRPM7 and miR-149 contributed to the proliferation and migration of human ASMCs induced by TGF- $\beta 1$. At the same time, this study also attempted to clarify the possible relationship between TRPM7 and miR-149. Our results manifested that the expression of miR-149 in human ASMCs was decreased after TGF-31 treatment. MiR-149 was involved in the regulation of proliferation and migration of human ASMCs. TRPM7 was a promising target gene of miR-149 in human ASMCs. Additionally, TRPM7 knockdown could inhibit the proliferation of human ASMCs, indicating that TRPM7 played an important regulatory role in airway remodeling. Therefore, we proved that miR-149 overexpression down-regulated the proliferation and migration of human ASMCs after TGF- $\beta 1$ pre-treatment by targeting TRPM7 in vitro.

\section{MATERIALS AND METHODS}

Experimental reagents. The experimental reagents were all purchased commercially. TGF- $\beta 1$, RIPA buffer and the experimental antibodies against TRPM7, $\beta$-actin, p-AKT, AKT, p-JNK, JNK, p-p38, p38, p-ERK1/2, ERK1/2 and GAPDH were purchased from Thermo Fisher Scientific, USA. The cell culture materials and fetal bovine serum (FBS) were obtained from Gibco Life Technologies (Grand Island, NY). 3-(4,5-Dimethylthiazol-2-yl)-2,5-diphenyltetrazolium bromide (MTT) was purchased from Chemicon (Temecula, CA, USA).

Cell culture. The cell line of ASMCs was purchased from ATCC (Manassas, VA, USA). Human ASMC cells were cultured in Dulbecco's modified Eagle's medium (DMEM) (Gibco, Rockville, MD, USA) containing 10\% FBS (Gibico, NY, USA Bio-Rad, CA, USA) and $1 \%$ penicillin $(100 \mu \mathrm{g} / \mathrm{mL}) /$ streptomycin $(100 \mathrm{U} / \mathrm{mL})$ at $37^{\circ} \mathrm{C}$ in an incubator with $5 \% \mathrm{CO}_{2}$.

Cell proliferation assay. Cell proliferation was conducted by MTT assay according to the standard protocol. MTT was purchased from Roche (Basel, Switzerland). Human ASMC cells were plated in a 96-well plate $\left(5 \times 10^{3}\right.$ cells per well $)$ and incubated with TGF- $\beta 1$ (1, 5 or $10 \mathrm{ng} / \mathrm{mL})$. After co-incubation for $24 \mathrm{~h}$ or $48 \mathrm{~h}$ each well was added with $20 \mu \mathrm{L}$ of $5 \mathrm{mg} / \mathrm{mL}$ MTT in PBS and co-incubated for another $4 \mathrm{~h}$ under $37^{\circ} \mathrm{C}$ in $5 \%$ $\mathrm{CO}_{2}$. Then $150 \mu \mathrm{L}$ DMSO was added to dissolve the formazan crystals. Viable cells were counted by measuring the OD (optical density) value using microreader at a wavelength of $450 \mathrm{~nm}$. Triple detection was performed in all experiments and average results were used to draw the growth curves.

RNA extraction and real-time quantitative reverse transcription-polymerase chain reaction $\mathrm{RT}$-qPCR (RT-qPCR). Total RNAs were extracted from cultured human ASMCs by using Trizol reagent (Thermo Fisher Scientific, USA) then converted into cDNA using the
RevertAid RT Reverse Transcription kit (Thermo Fisher Scientific, USA), which was performed according to the manufacturer's instructions. mRNA quantification was detected by RT-qPCR by using the RT-PCR kit (Takara Bio, Inc., Otsu, Japan) according to the standard protocols and GAPDH was used as the control gene. The relative primer sequences were prepared (Sangon, Shanghai, China) as follows:

-MiR-149-forward: 5'-GGCTCTGGCTCCGTGTCT'T -3' and miR-149-reverse: 5'-CAGTGCAGGGTCCGAGGTAT'T-3'.

-TRPM7-forward: 5'-AGTAATTCAACCTGCCTCAA-3' and TRPM7-reverse: 5'-ATGGGTATCTCTTCTGTTATGTT-3'.

-U6-forward: 5'-CAAATTCGTGAAGCGTTCCATA-3' and U6-reverse: 5'-AGTGCAGGGTCCGAGGTATTC-3'.

Each experiment was repeated three times. The foldchange for mRNA in tested samples relative to U6 was determined by the formula: $2^{-\Delta \Delta \mathrm{Ct}}$.

Cell transfection. MiR-149 mimic, negative control (NC mimic), miR-149 inhibitor (miR-149 inhibitor) and matched negative control (NC inhibitor) were synthesized by Shanghai Gena Pharma Corporation (Shanghai, China). Genomic DNA fragments of TRPM7 were prepared by the amplification of genomic DNA, cloned onto the pcDNA3.1-Luc reporter vector and verified by sequencing. The TRPM7 coding sequence was obtained by polymerase chain reaction (PCR) and cloned into the pcDNA3.1(+) plasmid. cDNA3.1-TRPM7 plasmid was constructed in our lab by inserting the ORF (open reading frame) of TRPM7 into a pcDNA3.1 plasmid. The shRNA sequences targeting TRPM7 gene are \#1: 5'-GCATAAATTCCT'TAC CAT'TC-3' and \#2: 5'-GCAAATGGAGT'TACCCAAAC-3', which were synthesized by GenePharma in Shanghai, China. All the transfection processes in this study were performed by Lipofectamine 2000 reagents (Invitrogen, Carlsbad, CA, U.S.A.) according to standard instructions.

Cell migration assay. Cell migration was estimated by transwell analysis, which was performed after cells were re-suspended $\left(1 \times 10^{5}\right.$ cells per $\left.\mathrm{mL}\right)$ in serum-free cell medium. Human ASMCs were co-incubated with TGF- $\beta 1(10 \mathrm{ng} / \mathrm{mL})$. After incubation at $37^{\circ} \mathrm{C}$ for $48 \mathrm{~h}$, the membranes were removed. At the same time, the human ASMCs on the upper side were scraped off. Finally, cells that migrated to the lower side of the membrane were fixed with $4 \%$ polyoxymethylene and stained with crystal violet. After drying, five visual fields were randomized under a light microscope to calculate the number of cells.

Luciferase reporter assay. To investigate the molecular interaction between miR-149 and TRPM7, luciferase reporter assay was performed in HEK-293T cells. The pmirGLO-TRPM7-3'UTR wild type (TRPM7 3'UTRW'T) and pmirGLO-TRPM7-3'U'TR mutant (TRPM7 3'UTR-MUT) were designed and purchased from Sangon (Shanghai, China). HEK-293T cells were planted in 12well plates with an amount of $1.5 \times 10^{5}$ per well. Then, $30 \mathrm{nM}$ miR-149 mimic or miR-NC was co-transfected with $300 \mathrm{ng}$ plasmid (TRPM7 3'UTR-WT or TRPM7 3'UTR-MUT) into HEK-293T cells by using transfection reagent Lipofectamine 2000 (Invitrogen) according to the standard protocol. After $24 \mathrm{~h}$ transfection, the relative luciferase activity was assessed with Dual- Luciferase Reporter Assay System (Promega, Madison, WI, USA).

Western blot analysis. Total proteins from the human ASMCs were lysed and extracted by RIPA Buffer (9800, Cell Signaling, Danvers, MA). The collected pro- 
A

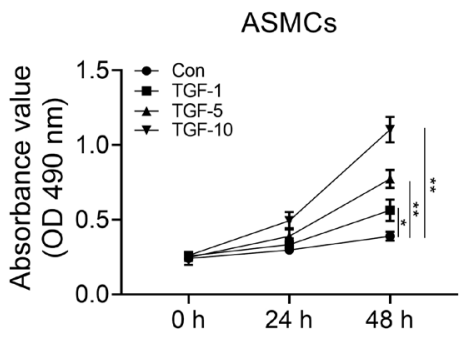

B

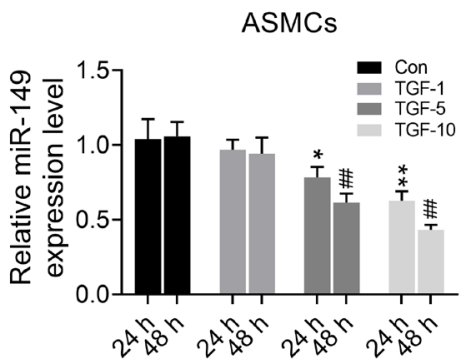

Figure 1. The expression of miR-149 in human ASMCs was decreased after TGF- $\beta 1$ treatment.

(A) MTT assay determined the cell proliferation of human ASMCs after TGF- $\beta 1$ treatment with different concentration (1, 5, and 10 ng/ $\mathrm{mL}$ ) for $24 \mathrm{~h}$ and $48 \mathrm{~h}$. (B) RT-qPCR analyzed the expression difference of miR-149 in ASMCs after TGF- $\beta 1$ treatment with different concentration $(1,5$, and $10 \mathrm{ng} / \mathrm{mL})$ for $24 \mathrm{~h}$ and $48 \mathrm{~h}$. All of the above results are presented as mean \pm S.D., ${ }^{*} p<0.05,{ }^{* *} p<0.01$ and ${ }^{\# \#} p<0.01$.

tein samples were then separated by SDS-PAGE gel electrophoresis. Subsequently, the gel was transferred to a polyvinylidene difluoride (PVDF) membrane and blocked with $5 \%$ milk for $1 \mathrm{~h}$ at room temperature. Then, the PVDF membranes were incubated overnight with anti-TRPM7 antibody, anti- $\beta$-actin antibody, antip-AKT/AKT antibodies, anti-p-JNK or JNK antibodies, anti-p-p38 or p38 antibodies, anti-p-ERK1/2 or ERK $1 / 2$ antibodies (Thermo Fisher Scientific, USA) at $4^{\circ} \mathrm{C}$. After washed by TBS-T solution for 3-5 times, the membranes were co-incubated with HRP-conjugated secondary antibody for $1 \mathrm{~h}$ at room temperature. Finally, the ECL detection reagents were used to detect the visualized immunocomplexes. Image Pro software was used to calculate the intensity of each blot.

Statistical analysis. All the experiments that performed in this project were repeated at least three times. The data was calculated using means and standard deviations. The software of SPSS 19.0 was used to analyze the data. ANOVA or $t$-test was applied to assess the significance $(p<0.05)$. Dunnett multiple comparison was used to test the difference between different groups. $p<0.05$ denotes statistically significant difference. $*$ indicates $p<0.05$, **indicates $p<0.01$ and $* * *$ indicates $p<0.001$.

\section{RESULTS}

\section{MiR-149 was down-regulated in human ASMCs after TGF- $\beta 1$ treatment}

In this study, the correlation between miR-149 expression and the TGF- $\beta 1$ treatment were investigated in human ASMCs (airway smooth muscle cells) in vitro. Firstly, MTT assay was conducted to determine the cell proliferation of human ASMCs after TGF- $\beta 1$ treatment with various concentrations (1, 5 and $10 \mathrm{ng} / \mathrm{mL}$ ) for $24 \mathrm{~h}$ and $48 \mathrm{~h}$, respectively. We found that human ASMCs treated with TGF- $\beta 1$ had a higher proliferation rate compared with control group. Moreover, the cell proliferation rate in TGF- $\beta 1$ group treated for $48 \mathrm{~h}$ was higher than that in $24 \mathrm{~h}$ treatment group, which demonstrated a time-dependent increase (Fig. 1A). In addition, the expression of miR-149 was also analyzed by RT-qPCR. The results showed that miR-149 expression was significantly down-regulated in human ASMCs treated with TGF- $\beta 1$.The expression level of miR-149 was negatively correlated with the TGF- $\beta 1$ concentration and the duration of stimulation (Fig. 1B). These results manifested that TGF- $\beta 1$ can promote cell proliferation and reduce the expression of miR-149 in human ASMCs.

\section{MiR-149 regulated proliferation and migration of human ASMCs induced by TGF- $\beta 1$ in vitro}

To investigate the effect of miR-149 on proliferation and migration in human ASMCs, cells that stably transfected with miR-149 mimic/inhibitor were constructed and verified by RT-qPCRRT-qPCR (Fig. 2A). The effect of miR-149 on the cell proliferation and cell migration in human ASMCs after TGF- $\beta 1$ treatment was detected by MTT assay and transwell assay, respectively (Fig. 2A and Fig. 2B). The experimental data showed that TGF- $\beta 1$ treatment could increase the cell proliferation rate and the number of migrated human ASMCs compared with untreated group $(p<0.01)$. Furthermore, based on TGF- $\beta 1$ treatment, the rate of cell proliferation and the number of migrating cells in miR-149 inhibitor-transfected cells was markedly increased $(p<0.01)$. While the miR-149 mimictransfected cells showed the contrary results. In conclusion, the above results showed that miR-149 inhibited the proliferation and migration of human ASMCs.

\section{TRPM7 is the target gene of miR-149 in human ASMCs}

On the basis of bioinformatics predication with Targetscan (http://www.targetscan.org), TRPM7 gene was found to be the potential gene of miR-149 (Fig. 3A). Then, luciferase reporter assay was conducted to determine the binding affinity of miR-149 to the wild-type of TRPM7 3'UTR in HEK-293T cells. The data indicated that the luciferase activity in miR-149 mimic-transfected HEK-293T cells was decreased significantly. But the overexpression of miR-149 cannot affect the luciferase activity of mutant TRPM7 3'UTR (Fig. 3B). Therefore, we suggested that the wild-type of TRPM7 3'UTR (TRPM7 3'UTR-wt) was the potential target gene of miR-149. To investigate how the miR-149 modulated the expression of TRPM7, RT-qPCR and western blot were conducted to analyze the relative mRNA expression and protein of TRPM7 in human ASMCs transfected with NC inhibitor/miR-149 inhibitor/NC mimic/miR-149 mimic. The results revealed that miR-149 mimic transfection group significantly decreased the mRNA and protein expression of TRPM7 in human ASMCs. While miR-149 inhibitortransfected group remarkably increased the expression of TRPM7 (Fig. 3C and Fig. 3D) RT-qPCR. Thus our results demonstrated that miR-149 regulated the expression of TRPM7 via a negative pattern. 
A

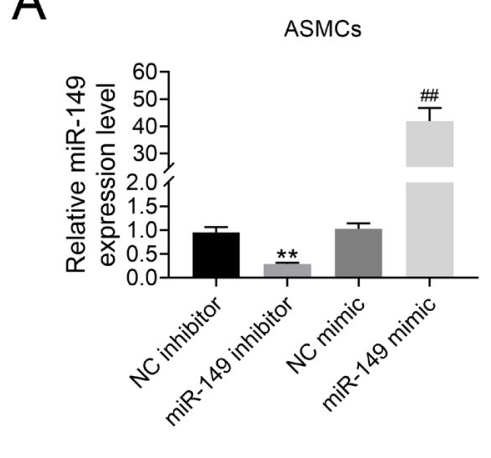

B

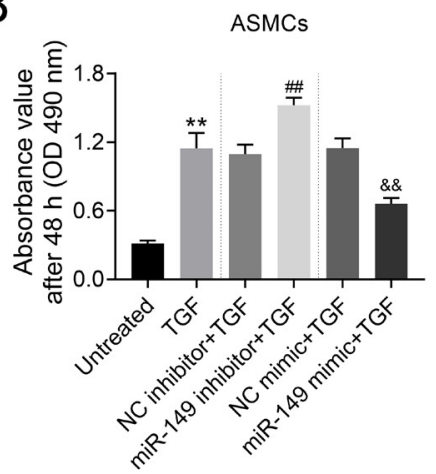

C

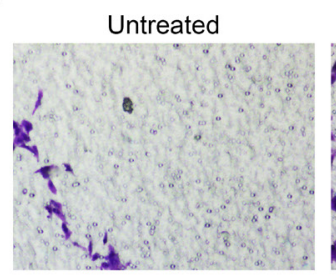

NC inhibitor+TGF

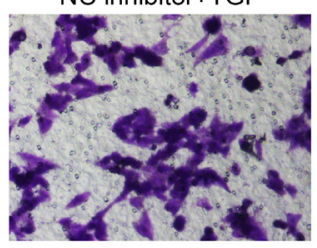

$\mathrm{NC}$ mimic+TGF

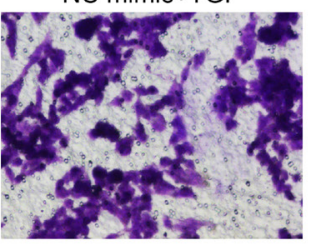

TGF

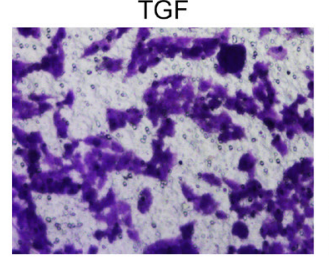

miR-149 inhibitor+TGF

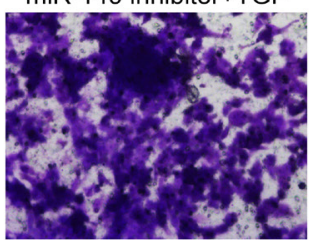

miR-149 mimic+TGF
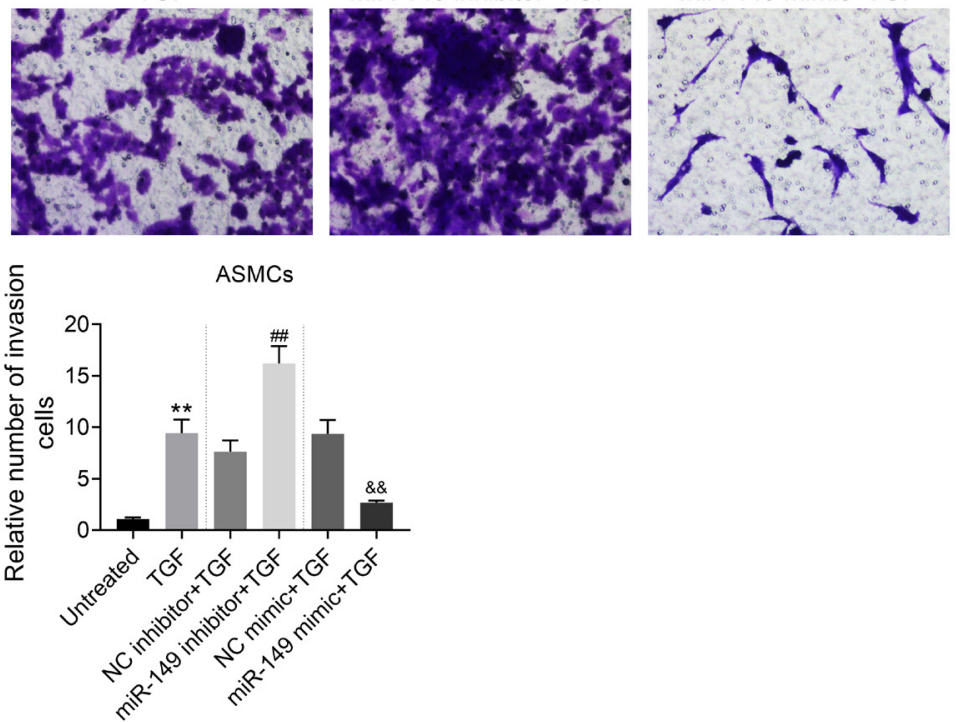

Figure 2. MiR-149 regulates proliferation and migration of human ASMCs induced by TGF- $\beta 1$ in vitro.

(A) RT-qPCR analyzed the relative expression levels of miR-149 in human ASMCs cells transfected with NC inhibitor/miR-149 inhibitor/NC mimic/miR-149 mimic. (B) MTT assay detected the cell proliferation of human ASMCs transfected with NC inhibitor/ miR-149 inhibitor/ NC mimic/miR-149 mimic after TGF- $\beta 1$ treatment for $48 \mathrm{~h}$. (C) Transwell assay detected the cell migration of human ASMCs transfected with NC inhibitor/miR-149 inhibitor/NC mimic/ miR-149 mimic after TGF- $\beta 1$ treatment for $48 \mathrm{~h}$. Results are presented as mean \pm S.D. All of the above results are presented as mean \pm S.D., ${ }^{*} p<0.05,{ }^{* *} p<0.01,{ }^{* \#} p<0.01$ and ${ }^{\& \&} p<0.01$.

\section{Knockdown of TRPM7 inhibits the activation of MAPKs pathway in vitro}

Human ASMCs transfected with shTRPM7\#1 and shTRPM7\#2 were used to explore the effects of TRPM7 on the activation of MAPK and AKT pathways. Western blot assay was conducted to analyze the expression difference of related protein (p-ERK1/2/ERK1/2, p-p38/ p38, p-JNK/JNK, p-AKT/AKT) levels in MAPK and AKT pathways in human ASMCs. The data showed that there was a significant down-regulation of phosphorylated proteins such as p-ERK1/2, p-p38 and p-JNK in human ASMCs that were transfected with hTRPM7\#1 and hTRPM7\#2. While the expression level of $\mathrm{p}-\mathrm{AK} T$ was not affected after knockdown of TRPM7 in human ASMCs. Therefore, we believed that TRPM7 played a key role in regulating the MAPK signaling pathway, and a knockdown of TRPM7 would significantly inhibit the activation of MAPK pathway in vitro (Fig. 4).

The interaction of miR-149 and TRPM7 affects the proliferation and migration induced by TGF- $\beta 1$ in human ASMCs

Previous studies have reported that TRPM7 were involved in the airway smooth muscle cell proliferation induced by TGF- $\beta 1$. Besides, miR-149 increased the risk of asthma in Chinese children (Hu et al., 2017)and in- 
A

Position 625-631 of TRPM7 3' UTR wt

hsa-miR-149-5p

TRPM7 3' UTR mut

B

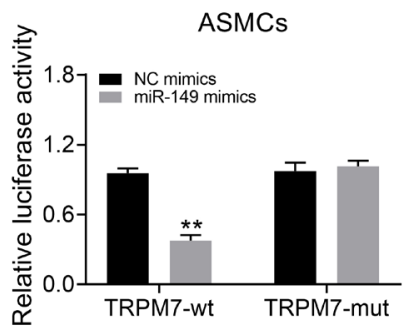

5' ...CCAUAUUUAAAgaUA--GAgccagu... 3'<smiles>C#Cc1c#cccc1C#C</smiles>

3' CCCUCACUUCUGUGCCUCGgUCU $5^{\prime}$

|| |

5' ...CCAUAUUUAAAGAUA--CUCGgUCU... 3'

\section{C}

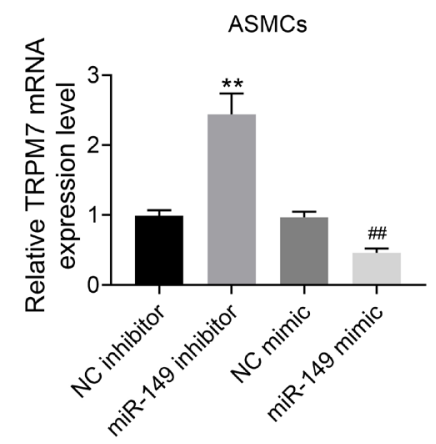

D
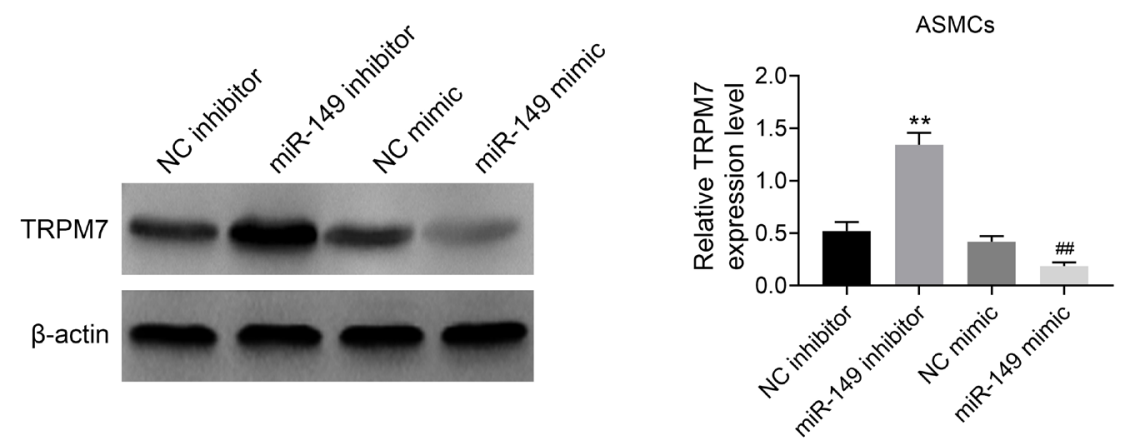

Figure 3. TRPM7 is the direct target of miR-149.

(A) In this diagram, the sequences of miR-149 binding sites complemented with TRPM7 3'-UTR (625-631). TRPM7 3'-UTR-wt represents the entire 3'UTR sequences of wild-type TRPM7, TRPM7 3'-UTR-mut represented the binding sites were mutated. (B) Luciferase reporter assays was performed in human ASMCs cells to detect the binding affinity of miR-149 to the TRPM7 3'UTR-wt or mut. (C) RT-qPCR analyzed the relative TRPM7 mRNA expression levels in human ASMCs cells transfected with NC inhibitor/miR-149 inhibitor/NC mimic/miR149 mimic. (D) Western blot analyzed the overexpression or inhibition of miR-149 in human ASMCs cells affected the TRPM7 protein levels. All of the above results are presented as mean \pm S.D., ${ }^{*} p<0.05,{ }^{* *} p<0.01$ and ${ }^{* \#} p<0.01$.

hibited cell proliferation and migration through MAPK pathway in various cancers (He et al., 2018; Zhi et al., 2018). Thus, we further explored whether miR-149 acted as a suppressor in MAPK pathway through targeting TRPM7 in asthma. The results of both MTT and transwell assays indicated that miR-149 could significantly inhibit proliferation and migration in human ASMCs after TGF- $\beta 1$ treatment, which could be reversed by transfecting cells with constructed TRPM7 overexpression plasmid (Fig. 5A, B). Western blot analysis also revealed a significant down-regulation of phosphorylated proteins such as p-ERK1/2, p-p38 and p-JNK in human ASMCs that were transfected with miR-149 mimics, which was recovered by TRPM7 overexpression. Moreover, an astonishing up-regulation of phosphorylated proteins such as p-ERK1/2, p-p38 and p-JNK in human ASMCs that had been transfected with TRPM7 was observed (Fig. 5C). We thus considered that miR-149 suppressed the MAPKs pathway through targeting TRPM7 in human ASMCs. In summary, we found that miR-149 suppressed the proliferation and migration of human ASMCs by targeting TRPM7 via regulating MAPKs pathway in vitro.

\section{DISCUSSION}

As a common chronic disease affecting the airways of the lungs and posing a serious threat to human health, asthma is characterized by remodeling of the airways, inflammatory reactions and hyperresponsiveness (Holgate, 2008). Although traditional therapies have made great progress, the lack of accurate biomarkers hinders the treatment of asthma (Tiotiu, 2018). Therefore, in order to treat asthma more effectively, people still need to further understand its pathogenesis to find effective therapeutic targets.

To date, many studies have shown that miRNAs play an important role in proliferation and migration of various diseases such as human malignancies (Benfey, 2003), asthma (Heffler et al., 2017), liver fibrosis (Schueller et 


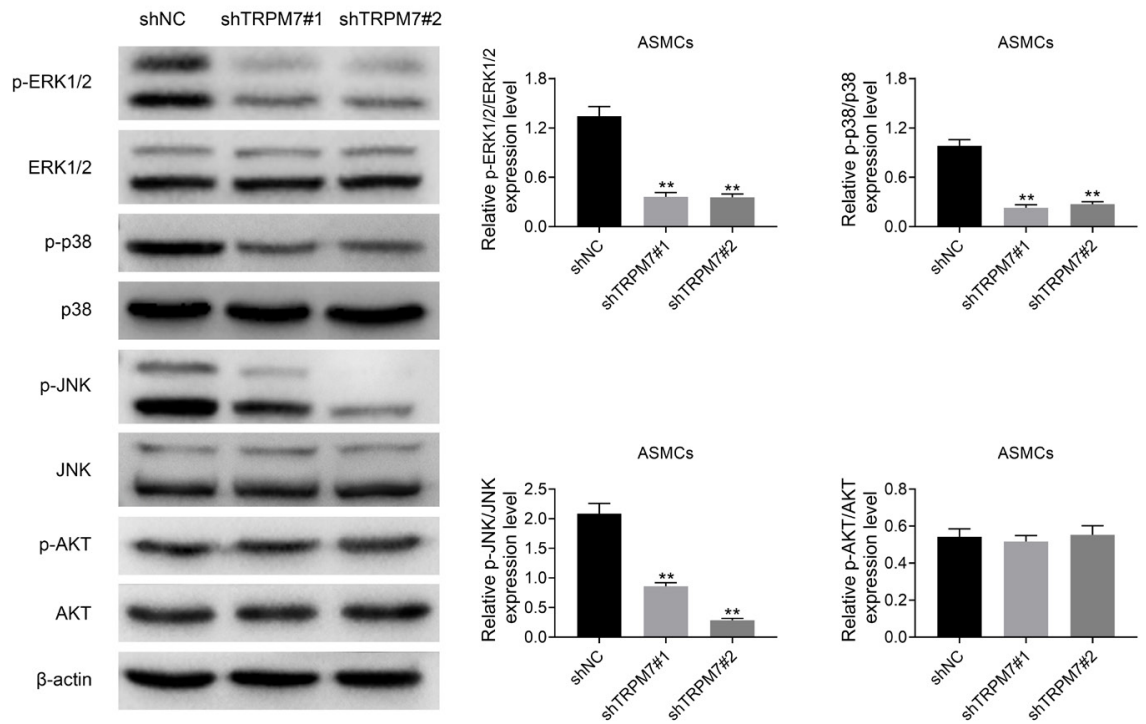

Figure 4. Knockdown of TRPM7 inhibits the activation of MAPKs pathway in vitro.

Western blot analyzed the expression difference of related protein (p-ERK1/2/ERK1/2, p-p38/p38, p-JNK/JNK, p-AKT/AKT) levels in MAPKs pathway in human ASMCs. All of the above results are presented as mean \pm S.D., ${ }^{* *} p<0.01$.

A
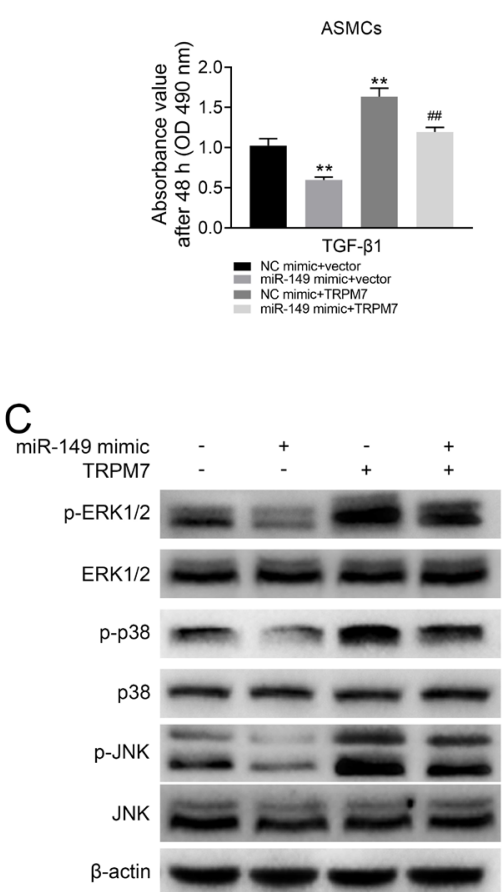

B
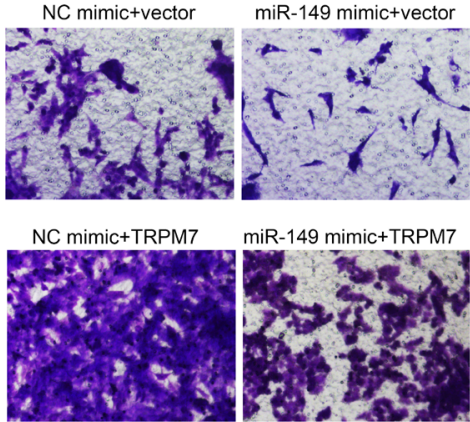
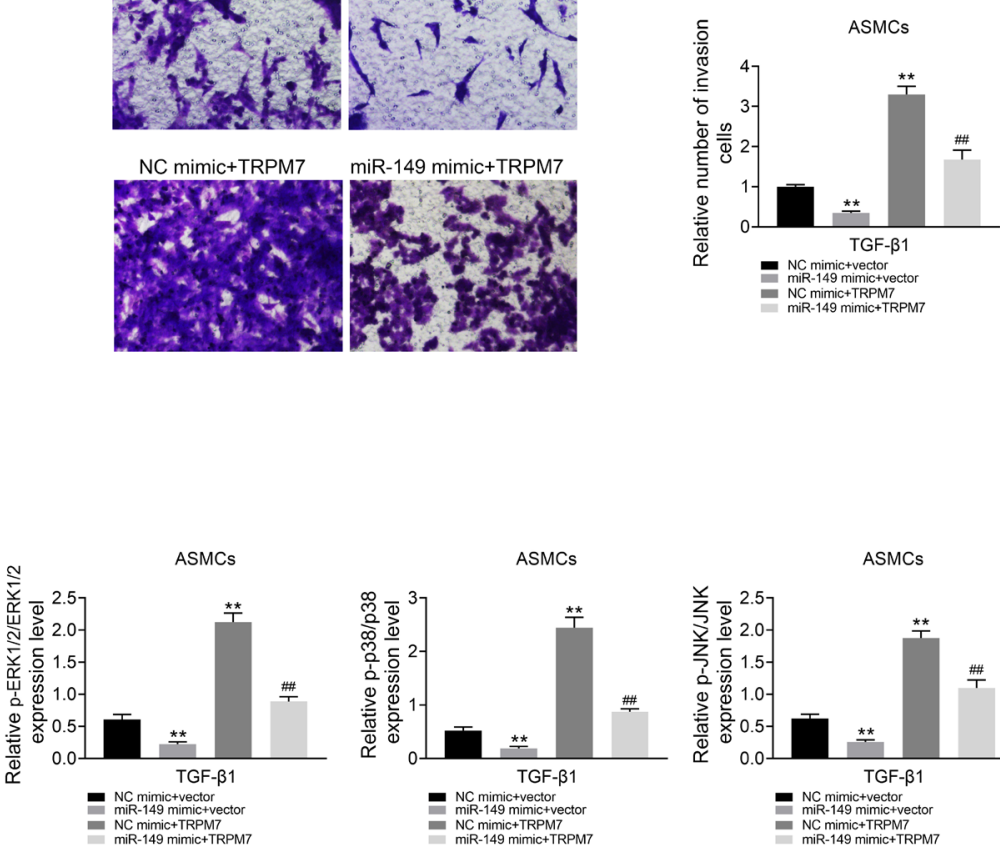

ASMCs
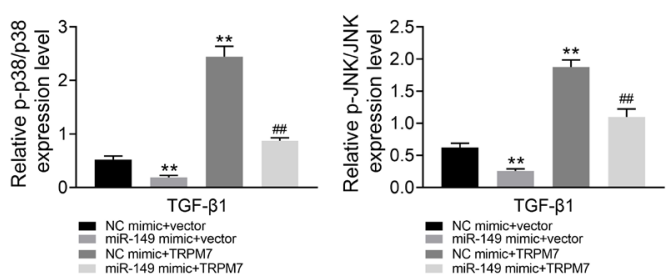

Figure 5. Effects of overexpressed miR-149 and TRPM7 on the proliferation and migration of human ASMCs induced by TGF- $\beta 1$ in vitro.

MTT assay (A) and Transwell assay (B) were used to evaluate the proliferation and migration of human ASMCs transfected with NC mimic+vector/miR-149+vector/NC mimic+TRPM7/miR-149 +TRPM7 after TGF- $\beta 1$ treatment. (C) Western blot analyzed the expression difference of related protein ( $p$-ERK1/2/ ERK1/2, p-p38/p38, $p$-JNK/JNK) levels of MAPKs pathway in human ASMCs that separately transfected with NC mimic+vector/miR-149+vector/NC mimic+TRPM7/miR-149 +TRPM7 after TGF- $\beta 1$ treatment for 48h. Results are presented as mean \pm S.D. $p$ values that less than 0.05 indicated statistical significance with representation ${ }^{*} p<0.05,{ }^{* *} p<0.01$ and ${ }^{* * *} p<0.001$. 
al., 2018)and so on. Previous studies have showed that miR-26a (Mohamed et al., 2010) and miR-150 (Perry et al., 2014) were up-regulated in people with asthma. MiR-149, a widely studied miRNA which has been shown to be involved in regulating cell proliferation and migration among different human diseases (He et al., 2018), attracted our attention. Multiple studies shown that the abnormal expression of miR-149 is involved in the regulation of tumor progression in gastric cancer (Wang et al., 2012) and breast cancer (Bischoff et al., 2014). Additionally, miR-149 has been found to be involved in suppressing the differentiation of bone marrow-derived mesenchymal stem cells (BMSCs) (Li et al., 2019). MiR-149 reduced the inflammatory response in osteoarthritis by inhibiting the activation of downstream signaling pathway (Chen et al., 2018). However, the key role of miR-149 in the growth of human ASMCs was still unclear. In this study, we found for the first time that overexpression of miR-149 inhibited the growth of human ASMCs that were treated with TGF- 31 in vitro. The molecular pathogenesis of miRNAs in the regulation of cancer metastasis is quite different. Several studies have found that miR-149 could down-regulate the tumor cell growth and migration by interacting with FOXM1 (Wang et al., 2012), PPM1F (Luo et al., 2015), EphB3 (Zhang et al., 2017), and GIT1 (Chan et al., 2014). Multiple evidences documented that miR-149 regulated the growth and invasion of cancer cells by targeting different downstream genes via different signaling pathways ( $\mathrm{He}$ et al., 2018; Zhi et al., 2018; Mao et al., 2019). However, the precise molecular pathogenesis of miR-149, as well as its direct target in asthma, remains unclear.

In this study, the results of RT-qPCR and western blot exhibited that the gene and protein expression levels of miR-149 were decreased in TGF- $\beta 1$ induced human ASMCs. Therefore, we assumed that the overexpression of miR-149 could inhibit the cell growth. MiR-149 overexpression was found to inhibit the cell growth and the cell migration in MTT and transwell assays, which could be reversed by downregulating the miR-149 expression. These results further verified the suppressive effects of miR-149 on cell proliferation and migration in human ASMCs treated with TGF- $\beta 1$.

TRPM7, a non-selective cation channel, is widely found in a variety of cancers and plays a key role in regulating cell survival, proliferation and invasion, as well as mediating different signaling pathways such as MAPK, ERK and PI3K (Gautier et al., 2016). Simultaneously, TRPM7 is found to be regulated by multiple miRNAs, thus affecting the cell proliferation and migration. For example, it was reposted that the overexpression of miR-543 (Liu et al., 2019) and miR-28-5p (Wan et al., 2019) inhibited the proliferation and migration by directly targeting TRMP7.

In this study, miR-149 was demonstrated to directly bind to the 3'UTR of a wild-type TRPM7. Furthermore, the overexpression of miR-149 could effectively inhibit TRPM7 expression in human ASMCs in vitro, which is consistent with the previous results. The overexpression or inhibition of miR-149 resulted in a decrease or an increase of TRPM7 expression. We thus believed that TRPM7 was a directly downstream target gene for miR-149.

Notably, other studies have reported that miRNAs directly targeted TRPM7 via suppressing the phosphorylation of MAPK pathway to inhibit tumor cells proliferation and migration (Meng et al., 2013; Chen et al., 2015). At present, our study also demonstrated the same mechanism for miR-149.
In conclusion, we found that miR-149 could suppress proliferation and migration in human ASMCs in vitro. Further studies revealed that miR-149 suppressed the cell proliferation and migration by targeting TRPM7 via MAPKs pathway. Taken together, this study strongly suggested that miR-149 might be an important biomarker for understanding the molecular pathogenesis of asthma, that could make an important contribution to the further development of novel therapies for asthma in the future.

\section{Competing interests}

The authors state that there are no conflicts of interest to disclose.

\section{Ethics approval}

Not applicable.

\section{Statement of Informed Consent}

Not applicable.

\section{Authors' contributions}

Zhengyu Zhu and Qian Zhang designed the study, supervised the data collection, analyzed the data, Liya Zhang and Ting Jiang interpreted the data and prepared the manuscript for publication, Yan Qian and Yun Sun supervised the data collection, analyzed the data and reviewed the draft of the manuscript. All the authors have read and approved the manuscript.

\section{Availability of data and materials}

All data generated or analyzed during this study is included in this published article.

\section{REFERENCES}

Bartel DP (2004) MicroRNAs: genomics, biogenesis, mechanism, and function. Cell 116: 281-297. https://doi.org/10.1016/s00928674(04)00045-5

Benfey PN (2003) Molecular biology: microRNA is here to stay. Nature 425: 244-245. https://doi.org/10.1038/425244a

Bischoff A, Huck B, Keller B, Strotbek M, Schmid S, Boerries M, Busch H, Muller D, Olayioye MA (2014) miR149 functions as a tumor suppressor by controlling breast epithelial cell migration and invasion. Cancer Res 74: 5256-5265. https://doi.org/10.1158/00085472.CAN-13-3319

Chan SH, Huang WC, Chang JW, Chang KJ, Kuo WH, Wang MY, Lin KY, Uen YH, Hou MF, Lin CM, Jang TH, Tu CW, Lee YR, Lee YH, Tien MT, Wang LH (2014) MicroRNA-149 targets GIT1 to suppress integrin signaling and breast cancer metastasis. Oncogene 33: 4496-4507. https://doi.org/10.1038/onc.2014.10

Chapman DG, Irvin CG (2015) Mechanisms of airway hyper-responsiveness in asthma: the past, present and yet to come. Clin Exp Allergy 45: 706-719. https://doi.org/10.1111/cea.12506

Chen Q, Wu S, Wu Y, Chen L, Pang Q (2018) MiR-149 suppresses the inflammatory response of chondrocytes in osteoarthritis by down-regulating the activation of TAK1/NF-kappaB. Biomed Pharmacother 101: 763-768. https://doi.org/10.1016/j.biopha.2018.02.133

Chen WL, Barszczyk A, Turlova E, Deurloo M, Liu B, Yang BB, Rutka JT, Feng ZP, Sun HS (2015) Inhibition of TRPM7 by carvacrol suppresses glioblastoma cell proliferation, migration and invasion. Oncotarget 6: 16321-16340. https://doi.org/10.18632/oncotarget. 3872

Faouzi M, Kilch T, Horgen FD, Fleig A, Penner R (2017) The TRPM7 channel kinase regulates store-operated calcium entry. J Physiol 595: 3165-3180. https://doi.org/10.1113/JP274006

Fleig A, Penner R (2004) The TRPM ion channel subfamily: molecular, biophysical and functional features. Trends Pharmacol Sci 25: 633-639. https://doi.org/10.1016/j.tips.2004.10.004

Gan L, Denecke B (2013) Profiling pre-microRNA and mature microRNA expressions using a single microarray and avoiding separate sample preparation. Microarrays (Basel) 2: 24-33. https://doi. org/10.3390/microarrays2010024 
Gautier M, Perriere M, Monet M, Vanlaeys A, Korichneva I, DhenninDuthille I, Ouadid-Ahidouch H (2016) Recent advances in oncogenic roles of the TRPM7 chanzyme. Curr Med Chem 23: 4092-4107. https://doi.org/10.2174/0929867323666160907162002

He Y, Yu D, Zhu L, Zhong S, Zhao J, Tang J (2018) miR-149 in Human cancer: a systemic review. J Cancer 9: 375-388. https://doi. org/10.7150/jca.21044

Heffler E, Allegra A, Pioggia G, Picardi G, Musolino C Gangemi, S (2017) MicroRNA Profiling in asthma: potential biomarkers and therapeutic targets. Am J Respir Cell Mol Biol 57: 642-650. https:// doi.org/10.1165/rcmb.2016-0231TR

Holgate ST (2008) Pathogenesis of asthma. Clin Exp Allergy 38: 872897. https://doi.org/10.1111/j.1365-2222.2008.02971.x

Hu D, Zhang Z, Ke X, Kang H, Hong S (2017) A functional variant of miRNA-149 confers risk for allergic rhinitis and comorbid asthma in Chinese children. Int J Immunogenet 44: 62-70. https://doi. org/10.1111/iji.12307

Kim S, Lee CH, Jin KN, Cho SH, Kang HR (2018) Severe asthma phenotypes classified by site of airway involvement and remodeling via chest CT scan. I Investig Allergol Clin Immunol 28: 312-320. https://doi.org/10.18176/jiaci.0265

Li Y, Yang F, Gao M, Gong R, Jin M, Liu T, Sun Y, Fu Y, Huang Q, Zhang W, Liu S, Yu M, Yan G, Feng C, He M, Zhang L, Ding F, Ma W, Bi Z, Xu C, Yuan Y, Cai B, Yang L (2019) miR-149$3 p$ Regulates the switch between adipogenic and osteogenic differentiation of BMSCs by targeting FTO. Mol Ther Nucleic Acids 17: 590-600. https://doi.org/10.1016/j.omtn.2019.06.023

Liu X, Gan L, Zhang J (2019) miR-543 inhibites cervical cancer growth and metastasis by targeting TRPM7. Chem Biol Interact 302: 83-92. https://doi.org/10.1016/j.cbi.2019.01.036

Lou W, Liu J, Gao Y, Zhong G, Chen D, Shen J, Bao C, Xu L, Pan J, Cheng J, Ding B, Fan W (2017) MicroRNAs in cancer metastasis and angiogenesis. Oncotarget 8: 115787-115802. https://doi. org/10.18632/oncotarget.23115

Luo G, Chao YL, Tang B, Li BS, Xiao YF, Xie R, Wang SM, Wu YY, Dong H, Liu XD, Yang SM (2015) miR-149 represses metastasis of hepatocellular carcinoma by targeting actin-regulatory proteins PPM1F. Oncotarget 6: 37808-37823. https://doi.org/10.18632/oncotarget. 5676

Mao F, Zhang J, Cheng X, Xu Q (2019) miR-149 inhibits cell proliferation and enhances chemosensitivity by targeting CDC42 and BCL2 in neuroblastoma. Cancer Cell Int 19: 357. https://doi.org/10.1186/ s12935-019-1082-9

Meng X, Cai C, Wu J, Cai S, Ye C, Chen H, Yang Z, Zeng H, Shen Q, Zou F (2013) TRPM7 mediates breast cancer cell migration and invasion through the MAPK pathway. Cancer Lett 333: 96-102. https://doi.org/10.1016/j.canlet.2013.01.031

Mohamed JS, Lopez MA, Boriek AM (2010) Mechanical stretch upregulates microRNA-26a and induces human airway smooth muscle hypertrophy by suppressing glycogen synthase kinase-3beta. J Biol Chem 285: 29336-29347. https://doi.org/10.1074/jbc.M110.101147
Ojiaku CA, Yoo EJ, Panettieri RA Jr (2017) Transforming growth factor beta1 function in airway remodeling and hyperresponsiveness. the missing link? Am J Respir Cell Mol Biol 56: 432-442. https://doi. org/10.1165/rcmb.2016-0307TR

Perry MM, Tsitsiou E, Austin PJ, Lindsay MA, Gibeon DS, Adcock IM, Chung KF (2014) Role of non-coding RNAs in maintaining primary airway smooth muscle cells. Respir Res 15: 58. https://doi. org/10.1186/1465-9921-15-58

Schueller F, Roy S, Vucur M, Trautwein C, Luedde T, Roderburg C (2018) The role of miRNAs in the pathophysiology of liver diseases and toxicity. Int J Mol Sci 19. https://doi.org/10.3390/iims19010261

Siddiqui S, Shikotra A, Richardson M, Doran E, Choy D, Bell A, Austin CD, Eastham-Anderson J, Hargadon B, Arron JR, Wardlaw A, Brightling CE, Heaney LG, Bradding P (2018) Airway pathological heterogeneity in asthma: Visualization of disease microclusters using topological data analysis. J Allergy Clin Immunol 142: 1457-1468. https://doi.org/10.1016/j.jaci.2017.12.982

Tiotiu A (2018) Biomarkers in asthma: state of the art. Asthma Res Pract 4: 10. https://doi.org/10.1186/s40733-018-0047-4

Wan J, Guo AA, Chowdhury I, Guo S, Hibbert J, Wang G, Liu M (2019) TRPM7 induces mechanistic target of Rap1b through the downregulation of miR-28-5p in glioma proliferation and invasion. Front Oncol 9: 1413. https://doi.org/10.3389/fonc.2019.01413

Wang Y, Zheng X, Zhang Z, Zhou J, Zhao G, Yang J, Xia L, Wang R, Cai X, Hu H, Zhu C, Nie Y, Wu K, Zhang D, Fan D (2012) MicroRNA-149 inhibits proliferation and cell cycle progression through the targeting of ZBTB2 in human gastric cancer. PLoS One 7: e41693. https://doi.org/10.1371/journal.pone.0041693

Weidner J, Malmhäll C, Rådinger M (2019) microRNAs in asthma pathogenesis - from mouse to man. J Transl Genet Genomics. https:// doi.org/10.20517/jtgg.2018.30

Yee NS (2017) Role of TRPM7 in cancer: potential as molecular biomarker and therapeutic target. Pharmaceuticals (Basel) 10. https://doi. org/10.3390/ph10020039

Zhang G, Liu X, Li Y, Wang Y, Liang H, Li K, Li L, Chen C, Sun W, Ren S, Zhu P, Zhang L (2017) EphB3-targeted regulation of miR-149 in the migration and invasion of human colonic carcinoma HCT116 and SW620 cells. Cancer Sci 108: 408-418. https://doi. org/10.1111/cas.13161

Zhao Z, Zhang M, Duan X, Chen Y, Li E, Luo L, Wu W, Peng Z, Qiu H, Zeng G (2018) TRPM7 Regulates AKT/FOXO1-dependent tumor growth and is an independent prognostic indicator in renal cell carcinoma. Mol Cancer Res 16: 1013-1023. https://doi. org/10.1158/1541-7786.MCR-17-0767

Zhi Y, Zhou H, Mubalake A, Chen Y, Zhang B, Zhang K, Chu X, Wang R (2018) Regulation and functions of MicroRNA-149 in human cancers. Cell Prolif 51: e12465. https://doi.org/10.1111/ cpr. 12465 